\title{
The pharmacodynamics of enoxaparin in percutaneous coronary intervention with precise rapid enoxaparin loading (PEPCI-PRE study)
}

\author{
Jack L. Martin · Edward T. A. Fry • \\ Todd Martin - Trevor H. Atherley • \\ Seth S. Martin · Marvin J. Slepian
}

Published online: 10 May 2009

(C) Springer Science+Business Media, LLC 2009

Erratum to: J Thromb Thrombolysis

DOI 10.1007/s11239-009-0326-2

In this article the Percent therapeutic value in the first column of Table 2 appear incorrectly. They appear correctly below.

Table 2 Pharmacodynamic data

\begin{tabular}{|c|c|c|c|c|c|}
\hline & $5 \min$ & $2 \mathrm{~h}$ & $4 \mathrm{~h}$ & $6 \mathrm{~h}$ & $8 \mathrm{~h}$ \\
\hline Percent therapeutic $(0.5-1.8 \mathrm{IU} / \mathrm{ml})$ & 76 & 100 & 100 & 100 & 100 \\
\hline $\begin{array}{l}\text { Anti-Xa levels IU/ml after enoxaparin } 30 \mathrm{mg} \text { IV followed } \\
\text { by } 1 \mathrm{mg} / \mathrm{kg} \mathrm{SC}(n=26)\end{array}$ & $0.62 \pm 0.23$ & $0.94 \pm 0.18$ & $1.00 \pm 0.17$ & $0.89 \pm 0.13$ & $0.78 \pm 0.23$ \\
\hline
\end{tabular}

The online version of the original article can be found under doi:10.1007/s11239-009-0326-2.

\section{J. L. Martin ( $ه)$}

Main Line Health, Sharpe-Strumia Research Foundation,

Bryn Mawr Hospital, 130 S. Bryn Mawr Ave., Bryn Mawr, PA 19010, USA

e-mail: MartinJ@mlhs.org

E. T. A. Fry

The Care Group, LLC, St. Vincent Hospital, Indianapolis, IN, USA

T. Martin

Main Line Health, The Lankenau Hospital, Wynnewood, PA,

USA
T. H. Atherley

Newark Beth Israel Medical Center, Newark, NJ, USA

\section{S. S. Martin}

Department of Medicine, Duke University Medical Center,

Durham, NC, USA

M. J. Slepian

Sarver Heart Center, University of Arizona, Tucson, AZ, USA 